\title{
Evaluative priming reveals dissociable effects of cognitive vs. physiological anxiety on action monitoring
}

\author{
Lien De Saedeleer, \& Gilles Pourtois
}

Department of Experimental Clinical \& Health Psychology, Ghent University, Ghent, Belgium

\author{
Correspondence : \\ Gilles Pourtois \\ Department of Experimental-Clinical and Health Psychology \\ Ghent University
}

Henri Dunantlaan 2

9000 Gent, Belgium

Phone: +3292649144

Email: gilles.pourtois@ugent.be 


\begin{abstract}
Performance monitoring enables the rapid detection of mismatches between goals or intentions and actions, as well as subsequent behavioral adjustment by means of enhanced attention control. These processes are not encapsulated, but they are readily influenced by affective or motivational variables, including negative affect. Here we tested the prediction that worry, the cognitive component of anxiety, and arousal, its physiological counterpart, can each influence specific processes during performance monitoring. In two experiments, participants were asked to discriminate the valence of emotional words that were preceded by either correct (good) or incorrect (bad) actions, serving as primes in a standard evaluative priming procedure. In Experiment $1(n=36)$ we examined the influence of trait worry and arousal. Additionally, we included a face priming task to examine the specificity of this effect. Stepwise linear regression analyses showed that increased worry, but not arousal, weakened the evaluative priming effect and therefore the rapid and automatic processing of actions as good or bad. By contrast, arousal, but not worry, increased posterror slowing. In Experiment $2(n=30)$ state worry was induced using an anagram task. Effects of worry on action monitoring were trait but not state dependent, and only evidenced when actions were directly used a primes. These results suggest a double dissociation between worry and arousal during performance monitoring.
\end{abstract}

Keywords: anxiety; worry; arousal; priming; action 


\section{Introduction}

Optimization of goal-directed behavior is considered to be an important aspect of normal functioning within everyday life, since it allows people to maximize their chances of achieving pursued goals. In this framework, action monitoring enables to swiftly evaluate the accuracy of actions and to correct or adjust behavior when actions happen to mismatch with goals or intentions (Simons, 2010). The monitoring of actions and more generally human behavior has been integrated in a framework of evaluative and executive control (Botvinick, Braver, Barch, Carter, \& Cohen, 2001; Jones, Cho, Nystrom, Cohen, \& Braver, 2002; Yeung, Botvinick, \& Cohen, 2004). More specifically, the dorsal anterior cingulate cortex (ACC) and surrounding medial prefrontal cortex underlie the swift monitoring of actions (Bush, Luu, \& Posner, 2000; Ridderinkhof, Nteuwenhuis, \& Braver, 2007; Taylor, Stern, \& Gehring, 2007). The function of this system is twofold: (1) it detects conflicts or response errors, and (2) initiates subsequently an attention orienting to these events, which triggers behavioral adjustments (Notebaert et al., 2009; Simons, 2010). The first process is indexed by (electro-) physiological changes such as heart rate deceleration and the generation of a specific event-related brain potential (ERP), named the errorrelated negativity (ERN) or error negativity (Ne; see Ullsperger, Fischer, Nigbur \& Endrass, 2014 for a recent overview). On a behavioural level, the second attention-related process is indexed by an elevated skin conductance response and post-error slowing (PES) in which individuals are slower at responding to trials following errors compared to hits (Hajcak, McDonald, \& Simons, 2003b; Notebaert et al., 2009).

In the past, research primarily reported that the detection of adverse events (incorrect actions/response errors) is accompanied by enhanced arousal and attention orienting. More recently, a series of studies by Aarts, De Houwer, and Pourtois (2012, 2013) went beyond earlier findings by showing that dedicated internal monitoring systems enable individuals to rapidly tag 
affective values (negative or positive) onto self-generated actions (incorrect or correct). More specifically, these researchers showed that response errors inadvertently committed during a speeded go/nogo task were automatically and rapidly associated with a clear negative valence while correct actions were marked by positive affect, with measurable effects of this emotional tagging of actions at the level of the ERN/CRN component. In Aarts et al. (2012), actions that were performed during this speeded go/nogo task, served as primes for a subsequent interleaved word evaluation task (in which participants were asked to categorize words as positive or negative). In accordance with a typical evaluative priming effect (Fazio, Sanbonmatsu, Powell, \& Kardes, 1986; Hermans, De Houwer, \& Eelen, 1994), it was hypothesized that if actions (generated during the speeded go/nogo task) are automatically evaluated in terms of valence, this process would influence the subsequent emotional evaluation of the word (second task). More specifically, they expected participants to be quicker (and/or better) in categorizing negative words after unwanted response errors compared to categorizing positive words after these same incorrect actions. Furthermore, it was expected that the opposite effect would occur after correct responses (i.e., hits). Experimental support was provided for the notion that actions are swiftly evaluated along a genuine negative-positive valence dimension. In a follow-up study, Aarts et al. (2013) provided evidence for the involvement of the ERN/Ne and CRN (correct-related negativity) component in this process. Moreover, in the study by Aarts et al. (2012) the distinction between the rapid affective evaluation of actions and the subsequent PES was indirectly supported by the differential modulatory effect trait anxiety had on these two components. While higher levels of trait anxiety were negatively correlated with the size of the evaluative priming effect, no such or opposite relationship was found between trait anxiety and PES. Altogether, these findings suggest that there is an emotional component involved in the 
processing and monitoring of actions, and that this emotional component might be altered in (trait) anxiety or negative affect selectively.

The present study seeks to clarify and extend the results obtained previously by Aarts et al. (2012). More specifically, in their study, Aarts et al. (2012) found that trait anxiety undermined the affective tagging of actions, when levels of anxiety were measured, following standard practice, using the Spielberger State-Trait Anxiety Inventory (STAI; Defares, van der Ploeg, \& Spielberger, 1979). However, the STAI has limited discriminant value (Bishop \& Forster, 2013) and is therefore poorly sensitive to identify which specific component of anxiety might actually influence the affective tagging of actions. Accordingly, the first aim of this study was to identify which component of anxiety likely influences the affective tagging of actions. Anxiety can be divided at least into two subcomponents, namely a cognitive and a physiological component. Using hierarchical cluster analyses (carried out on questionnaire and fMRI data), Bijsterbosch, Smith, Forster, John, and Bishop (2014) recently confirmed the dissociation between a cognitive component (consisting of the Neuroticism scale from the Eysenck Personality Questionnaire, Eysenck, Eysenck \& Barrett, 1985; Penn State Worry Questionnaire, PSWQ, Meyer, Miller, Metzger, \& Borkovec, 1990; and STAI trait, Defares et al., 1979) and a physiological (arousal-related) component of anxiety (consisting of the Mood and Anxiety Symptom Questionnaire-anxious arousal subscale, Clark \& Watson, 1991; and the Anxiety Sensitivity Index, Peterson \& Reiss, 1987). Likewise, earlier electrophysiological results and models (see Moser, Moran, Schroder, Donnellan, and Yeung (2013) for a recent overview) already emphasized the distinction between worry and arousal when considering effects of anxiety on action monitoring. Anxious apprehension is usually defined as the propensity to engage in verbal rumination and/or worry when dealing with ambiguous future threats. By comparison, anxious arousal refers to the physiological component (hyperarousal) and somatic 
tension elicited by clear and current threats. Noteworthy, Moser et al. (2013) provided evidence for an association between anxious apprehension (worry) and an enhanced ERN component, with no such link found with anxious arousal, suggesting that worry (but not arousal) might actually play a predominant role in abnormal early error monitoring processes typically observed in high anxious individuals (Endrass \& Ullsperger, 2014; Koban \& Pourtois, 2014).

An older dominant model accounting for modulatory effects of anxiety on cognition stated that worry might reflect the occupation (or hijacking) of resources that would otherwise be allocated to the control of attention (M. W. Eysenck, 1979). More recently, several models have confirmed that worry, which can be conceived as a component of mind-wandering, may shift attention away from external and task-relevant stimuli, which in turn might impair behavioral performance (Barron, Riby, Greer, \& Smallwood, 2011). In line with this assumption, Forster, Elizalde, Castle, and Bishop (2015) recently reported, using fMRI, that during blocks of (unwanted) error commission, increased anxiety or worry-related dorsolateral prefrontal cortex activity could reflect spontaneous shifts of thoughts away from the task at hand to self-relevant information. Hence, worry appears to play a detrimental role during attention control, and presumably action monitoring too. As advocated by Borkovec, Alcaine, and Behar (2004) in their Avoidance Theory of Worry, worry may actually dampen arousal and subsequently interfere with (the normal) emotional processing of upsetting events, which might in turn lead to an extinction or suppression of responses to emotionally evocative stimuli. On the other hand, Mogg, Weinman, and Mathews (1987) suggest with their vigilance avoidance hypothesis that in order to avoid accurate evaluation of aversive events or habituation of them, anxious individuals first have the tendency to quickly identify potentially threatening events. Interestingly, this explanation might potentially explain why higher levels of anxiety were eventually associated with a weaker action-based evaluative priming effect (Aarts et al., 2012). More specifically, if individuals with 
high levels of anxiety are faster in identifying threatening events, it is possible that for them response errors (prime, which are negatively connoted) are processed differentially or to a larger extent than participants with a lower level of trait anxiety, hindering in turn the processing of the subsequent target stimulus (i.e., emotional word) and decreasing priming between these two events. In this latter framework, the worry component of anxiety would impair the affective processing of actions, and therefore lead to a lower priming or transfer effect to the external emotional words following these self-generated actions. Accordingly, in light of the evidence reviewed here above, we surmise that worry (defined as a trait) could be the main or critical component accounting for a diminished action-based evaluative priming effect in high anxious individuals. This conjecture lies at the heart of this study.

To test this prediction, we conducted two separate experiments. In Experiment 1, we used the task previously validated by Aarts et al. $(2012,2013)$ in a large sample of healthy adult participants, but we measured not only trait anxiety broadly speaking (using the STAI), but also levels of worry and arousal, separately. Following standard practice (Moser et al., 2013), the Penn State Worry Questionnaire (PSWQ; Meyer, Miller, Metzger, \& Borkovec, 1990) was used in combination with the subscale Anxious Arousal of the Mood and Anxiety Symptom Questionnaire (MASQ-AA; Clark \& Watson, 1991), to titrate worry and arousal, respectively. Moreover, because previous research already showed that skin conductance responses predicted the PES (Hajcak, McDonald, \& Simons, 2003a), we additionally surmised that anxious arousal (Critchley, Elliott, Mathias, \& Dolan, 2000; Lang, Greenwald, Bradley, \& Hamm, 1993), but not worry, could influence (i.e., augment) PES in our experiment. In other words, while we hypothesized that worry could interfere with the rapid affective tagging of actions selectively, we reckoned that anxious arousal could increase PES specifically, revealing a functional dissociation between these two components of anxiety during action monitoring. 
Further, because previous studies already found blunted priming effects associated with (trait) anxiety irrespective of action monitoring (Berner \& Maier, 2004; Glaser \& Banaji, 1999; Maier, Berner, \& Pekrun, 2003), we also included a control task in Experiment 1 where a standard cue-target priming sequence (with external stimuli) was used. As external visual primes, we used angry and happy faces extracted from the Karolinska Directed Emotional Faces set (AKDEF; Lundqvist, Flykt \& Öhman, 1998). Visual targets were the exact same emotional words as used during the main task. Hence, we could eventually compare the processing of the same visual targets (i.e., emotional words), when they were immediately preceded either by actions or emotional faces. This way, we could assess whether modulatory effects exerted by worry were specific to the action-based priming effect, or they could also extend to cases where a standard evaluative priming effect was used.

In Experiment 2, we used the same action-based evaluative priming task as in Experiment 1 , and induced worry by means of an orthogonal anagram task. Because state anxiety (or more specifically worry in the present case) is tightly related to trait anxiety (M. W. Eysenck, Derakshan, Santos, \& Calvo, 2007), the question arose whether state worry could yield similar interference effects during action monitoring than trait worry. For example, Egloff and Hock (2001) showed that trait and state anxiety had comparable influence on the emotional Stroop task. Furthermore, Williams, Mathews, and Hirsch (2014) pointed out that worry manipulations (and therefore state worry) can have deleterious effects on cognitive functions such as attention. Hence, in Experiment 2, we explored whether state worry influenced the rapid affective tagging of actions, like trait worry did (Experiment 1).

\section{Experiment 1}

\section{Method}


Participants. Thirty-six participants took part in Experiment 1. Seven participants were excluded from the subsequent statistical analysis of the action priming task, since they did not commit a sufficient number of False Alarms (i.e., minimum 10) to compute reliable accuracy or RT estimates for each condition separately (i.e., positive words following False Alarms vs. negative words following False Alarms). The remaining participants were mainly female (22 women, 7 men $)$ and had a mean age of 25 years $(S E M=1.25$, range $=18-46)$. All participants were native Dutch speakers. Participants were asked to complete two tasks (Action affective priming task vs. Face affective priming task) on two different occasions, separated by a week. As compensation, they received 20 euros.

\section{Stimuli and instruments.}

\section{Action affective priming task.}

Go/noGo task. Participants were seated in front of a computer monitor that consisted of a white homogenous background. Against that background, an arrow (subtending 11.4 x 0.05 of visual angle at a $60 \mathrm{~cm}$ viewing distance) was presented either upward or downward. In the initial state, the arrow had a black color that could subsequently turn either turquoise or green. The luminance of both turquoise and green were matched. Different combinations in orientation and color served as cues in the Go/noGo task.

Evaluative categorization task. Words had to be categorized as either being positive or negative. More specifically, 30 positive and 30 negative words were selected from the Dutch affective rating list of Hermans and De Houwer (1994) that could either be nouns or adjectives (as used in Aarts et al., 2013). More specifically, in Hermans and De Houwer (1994), undergraduate students rated these words on a 7-point likert scale. Moors et al. (2013) replicated the study of Hermans and De Houwer (1994) with a present-day sample of students and found that ratings of their recent study correlated highly with the ratings of Hermans and De Houwer 
(1994). Based on the ratings of Moors et al. (2013), for the present study mean valence and arousal scores were computed for words belonging to the positive and negative group. In case of valence, positive words were significantly rated as more positive $(M=5.70, S E M=0.13)$ on a 7 point likert scale compared to negative words $(M=2.13, S E M=0.10), t(58)=-22.17, p<0.001$, $95 \%$ CI $[-3.89,-3.25], d=-5.72$. In case of arousal, no significant difference was found between positive $(M=4.73, S E M=0.13)$ and negative $(M=4.56, S E M=0.20)$ words, $t(58)=0.78, p=$ $0.44,95 \%$ CI $[-0.29,0.67], d=0.20$. The positive and negative words did not differ either on the familiarity dimension, $\mathrm{t}<1$, nor with respect to the number of letters, $t<1$.

Face affective priming task.

Face discrimination task. Rectangles (black outline with a white fill, which served as cues) were presented in the center of the screen on a white homogenous background and were followed by pictures of faces (both $200 \times 271$ pixels) that had to be either categorized as positive or negative. The pictures were derived from the Karolinska Directed Emotional Faces set (AKDEF; Lundqvist et al., 1998). The pictures of 48 different actors/identities (24 female, 24 male) were selected and shown using a frontal viewpoint. Only angry and happy expressions were used.

Evaluative categorization task. The exact same words were used as in the evaluative categorization task that was associated with the go/nogo task (i.e., action affective priming task).

\section{Questionnaires.}

Penn State Worry Questionnaire. A Dutch version of the Penn State Worry Questionnaire (PSWQ) was used. The PSWQ measures the intensity and frequency of worry through a 16 item self-report questionnaire. Each item consists of a particular statement of which individuals need to indicate to what extent it is considered a typical behaviour. In doing so, participants have to rate the items on a 5-point Likert scale ranging from 1 -Not at all typical to 5 -Very typical. This 
questionnaire has shown to have a high test-retest reliability (ranging from .74 to .93; Molina \& Borkovec, 1994) and assesses a unidimensional construct with a high internal consistency (coefficient $\alpha$ of .91; see Meyer et al., 1990). Furthermore, convergent and discriminant validities of the scale are deemed high and are usually provided by correlations between the PSWQ and measures of emotional control, anxiety and/or depression (Brown, Antony, \& Barlow, 1992).

$M A S Q-A A$. Anxious Arousal is a subscale of the Mood and Anxiety Symptom Questionnaire (Clark \& Watson, 1991). The entire questionnaire consists of 90 items that are related to symptoms including anhedonic depression, general distress and anxious arousal. Participants indicated on a 5-point Likert scale ranging from 1- Not at all to 5-Extremely, to what extent they experienced those described symptoms during the past week, including the day of testing. More specifically, the Anxious Arousal subscale tackles the physiological component of anxiety. The MASQ has been validated by previous studies (Keogh \& Reidy, 2000; Watson et al., 1995).

STAI. The Dutch version of the Spielberger State-Trait Anxiety Inventory (Defares et al., 1979) was used. Both the state and trait version were used. Both versions consist of 20 items with which participants needed to indicate on a scale going from 1- Not at all to 4- Extremely to what extent they agree.

Procedure. Participants were informed that the experiment consisted of two sessions separated from each other by exactly one week. Within these two sessions, they were instructed to perform either the face affective priming task or the action affective priming task. The battery of questionnaires administered depended on the session (see Figure 1). The order of the sessions was counterbalanced across participants. Additionally, two versions of each task were made in 
which the response mapping was reversed. At the beginning of the experiment, participants gave their written informed consent. The study was approved by the local ethics committee.

Action affective priming task. Participants performed a standard speeded Go/noGo task (Vocat, Pourtois, \& Vuilleumier, 2008) interleaved with a visual word categorization task (see Figure 1). Actions performed during the speeded Go/noGo task actually served as primes whereas words were used as targets in analogy with a conventional prime-target sequence during evaluative priming. Each trial started with a fixation cross that lasted for $500 \mathrm{~ms}$. Afterwards, a black arrow, oriented up or down, was presented at the position previously occupied by the fixation cross. After a variable interval ranging from $1000 \mathrm{~ms}$ to $2000 \mathrm{~ms}$, the black arrow turned either green or turquoise while its (in-plane) orientation could remain identical or shift in the opposite direction compared to the initial black arrow. When the black arrow turned green and the orientation remained unchanged, participants were instructed to press a pre-defined button of the response box as fast as possible with the index finger of their left hand (Go trials). However, participants had to withhold responding when either the arrow became green but changed orientation, or when the arrow became turquoise and kept its initial orientation, enabling two noGo trial types. Instructions emphasized both speed and accuracy, such that not only accuracy, but also the actual speed was later evaluated as being either correct or incorrect. Since a sufficient number of response errors was required for subsequent analyses, participants were encouraged to respond fast (i.e., to adopt an impulsive response mode, while striving to be accurate as well). They were told that correct but too slow responses would be processed as incorrect events (like response errors), further emphasizing the need to be fast (besides being correct). More specifically, information regarding performance was provided in the form of trial by trial feedback and the presentation of cumulative accuracy (based on trial history) shown in the upper right corner of the screen during every trial. 
To determine whether a correct hit was fast enough or not, the experiment consisted of three calibration blocks (in which reaction time (RT) cutoffs were calculated) and six test blocks in which these RT cutoffs (depending on the calibration block) were directly used as response deadline (one calibration block always preceded two consecutive test blocks). More specifically, in each calibration block, a mean RT was calculated based on twenty Go trials where speed was emphasized too. Participants received negative feedback ("Too Slow") during the (subsequent) test blocks if their speed was slower than this (arbitrary) RT limit. Because several calibration blocks ( $\mathrm{n}=3$ ) were included (each time with a RT estimate computed for 20 trials) and the response deadline was adjusted each time accordingly, these RT cutoffs were not influenced by outliers (in terms of speed). Thus, speed during test blocks was evaluated using an individually calibrated RT limit computed during a yoked calibration block that always preceded a pair of test blocks. This limit was calculated and updated three times in total (before Blocks 1 and 2 i.e., Session 1; before Blocks 3 and 4 i.e., Session 2; and before Blocks 5 and 6 i.e., Session 3). This procedure allowed us to deal with unspecific learning effects over time and maintain a high number of response errors throughout the experimental session. For the first session, the upper limit was set to $70 \%$ of the mean RT from the first calibration block. For the two subsequent sessions, this upper limit was updated and set to $80 \%$ of the mean RT during the respective calibration block. Hence, this procedure required participants to respond at least 30\% faster (first session) or $20 \%$ faster (second and third sessions) on Go trials than their average speed during the respective training block. This procedure further allowed us to distinguish between Fast Hits (i.e., correct responses on Go trials that were made faster than the individually-titrated RT limit) and Slow Hits (i.e., correct responses on Go trials that were made slower than the RT limit). Participants were never informed about the underlying algorithm used to set and update this adaptive RT cutoff. Errors were formally defined as overt responses on noGo trials (False 
Alarms), while correct inhibitions corresponded to correctly withheld responses on the same noGo trials. Three hundred milliseconds after an action was executed, a single target word that was randomly selected from a list containing 60 evaluative words (30 negative and 30 positive; see stimuli section), was presented. Accordingly, repetitions of words occurred but these repetitions were on average balanced and fully non-predictable. For correct inhibitions, the target word was presented $1800 \mathrm{~ms}$ after the presentation of the colored arrow. Participants were instructed to categorize the valence of the target word (positive or negative) as fast and as accurately as possible by pressing one of two predefined keys of the response box using their dominant hand. Two versions of the task were made in which the response mapping of the word categorization task was reversed. Hence, the evaluative word categorization task was executed with a different effector than the Go/noGo task. The target word remained on the screen until the participant responded or $3000 \mathrm{~ms}$ elapsed. In order to balance the presentation of positive vs. negative words following Fast Hits, Slow Hits, Correct Inhibitions, and False Alarms, the target word that was presented following an action was selected randomly on each trial. After the word categorization, participants received feedback informing them about their accuracy for the two consecutive tasks. The feedback for the Go/noGo task indicated whether the performed action was correct (and fast enough), incorrect or too slow, while the feedback for the word categorization could be either correct or incorrect. Both feedback signals remained on the screen for $1000 \mathrm{~ms}$.

After a practice phase including 24 trials, the experiment was divided into three sessions, each starting with a training block (containing 28 trials: 20 Go and 8 noGo trials), followed by two test blocks (each containing 72 trials: 48 Go and 24 noGo trials). Note that participants were unaware that training blocks were actually used as calibration blocks to compute the RT limit used during the two following test blocks. Trial presentation was randomized within blocks. 
Between blocks, a small break (no longer than $5 \mathrm{~min}$ ) was introduced. The whole task included 540 trials and lasted on average $50 \mathrm{~min}$. Stimulus presentation and response recording were controlled using E-prime software (V2.0., http://www.pstnet.com/products/e-prime/). After the action affective priming task, participants were invited to fill in the questionnaires.

Face affective priming task. Participants performed a face discrimination task interleaved with an evaluative word categorization task (see Figure 1). In the first task they were instructed to perform a go/nogo task in analogy with the speeded go/nogo task outlined here above. More specifically, depending on the block, they had to respond either to positive or negative faces only, and hence to withdraw from responding if the other facial expression was shown. The face was considered to serve as a prime for the consequent target word evaluation. Similar to the procedure of the Action affective priming task, each trial started with a fixation cross that lasted on the screen for $500 \mathrm{~ms}$. Afterwards, an upward rectangle serving as a cue was shown for a random time ranging from $1000 \mathrm{~ms}$ to $2000 \mathrm{~ms}$. After this time, the cue was replaced by a positive or negative face that was presented in the center of the screen, which could stay for a maximum duration of $1500 \mathrm{~ms}$ or until the participant responded. Depending on the block, participants were instructed to respond either only to positive or negative faces by pressing a pre-defined button of the response box with the index finger of their left hand (Go trials). If the other facial expression was shown, participants had to withhold responding (noGo trials). Participants were instructed to be both as fast and as accurate as possible. If the reaction time was above $400 \mathrm{~ms}$ on a particular trial, this was fed back as being too slow. This limit was used to ensure that the face discrimination task was performed with a response deadline, similarly to the Action affective priming task. However in the case of the (control) Face Priming Task (FPT), there was no need to collect a high number of response errors and therefore speed was not as emphasized as in the Action-based Priming Task (APT). Therefore no calibration phases were included and a uniform 
cutoff of $400 \mathrm{~ms}$ was used throughout to make sure that participants responded as fast as possible (with a high amount of correct responses collected). This cutoff of $400 \mathrm{~ms}$ was based on pilot testing that yielded the expected results (despite some inherent inter-individual variability regarding RT speed). Three hundred ms after responding to the face, a target word was randomly selected from a list containing 60 evaluative words (30 negative and 30 positive; see stimulus section) and presented. Accordingly, repetitions of words occurred but these repetitions were on average balanced and fully non-predictable. If participants did not respond to the face, the target occurred $1800 \mathrm{~ms}$ after the onset of the face. Participants were instructed to categorize the valence of the target word (positive or negative) as fast and as accurately as possible by pressing one of two predefined keys of the response box using their right hand. Two response mappings were applied for the word evaluation task, in such a way that some participants were randomly assigned to use their right index for evaluating negative words and their right middle finger for evaluating positive words. In the other version, this specific stimulus-response mapping was reversed. Hence, the evaluative word categorization task was executed with a different effector than the face discrimination task. The target word remained on the screen until the participant responded or $3000 \mathrm{~ms}$ elapsed. Each positive and negative word was preceded by a positive or negative face an equal amount of times. After the word categorization, participants received feedback concerning their accuracy for the two consecutive tasks. The feedback for the face discrimination task indicated whether the performed action was correct (and fast enough), incorrect or too slow, while the feedback for the evaluation of the words could be either correct or incorrect. Both feedback signals remained on the screen for $1000 \mathrm{~ms}$.

Since there were two types of blocks (with either the positive or negative faces to respond to), two practice blocks were introduced. In one block participants were instructed to respond to positive faces but not to negative faces. In the other block they had to respond to negative faces 
and ignore positive faces. Each practice block contained 12 trials. The order of the two blocks was randomly determined. The task included six experimental blocks. In three blocks participants had to respond to positive faces and withhold responding to negative faces, whereas in the other three blocks they had to respond to negative faces and withhold responding to positive faces. Each experimental block contained 72 trials, of which 48 were Go trials and 24 were noGo trials. The order of these experimental blocks was randomized, as was trial presentation within blocks. Participants could take short breaks between blocks. On average the task lasted around 35 minutes and included in total 456 trials. Stimulus presentation and response recording were controlled using E-prime software (V2.0., http://www.pstnet.com/products/e-prime/)

\section{Analyses of Behavioral Data}

\section{Action priming task.}

Outliers. Trials with RTs shorter than 150 ms or longer than 500 ms during the Go/noGo task were discarded (see Aarts et al., 2012). Regarding False Alarms, 10\% of trials were removed, whereas $9 \%$ of Fast Hits were removed. Across all trial type, the removal amounted $6 \%$ in total. For the subsequent evaluative categorization task, trials were excluded if RT exceeded $2.5 \mathrm{SD}$ above or below the mean reaction time computed per condition (Negative words: $4 \%$, Positive words: 4\%). Using these criteria on a group level, the data of five participants (two outliers based on RT and three other based on accuracy) were removed from the subsequent statistical analyses. After exclusion, on average 34 trials and 32 trials were included to compute mean RTs for negative words and positive words after False Alarms, respectively. In the case of words following Fast Hits, on average 54 trials and 55 trials were included for negative words and positive words, respectively ( $\mathrm{n}=27$ participants). For accuracy ( $\mathrm{n}=26$ participants), on average 35 trials and 35 trials were included to compute mean performance for negative words and positive 
words after False Alarms, respectively. In case of words following Fast Hits, on average 56 trials and 57 trials were included for negative words and positive words, respectively

Go/noGo task. Accuracy and RTs were analyzed separately using paired samples T-tests. All data were analyzed using SPSS (version 20).

Evaluative categorization task. Accuracy and RTs (for correct responses) were analyzed separately using a repeated measures ANOVA with (i) the valence of the target word (either positive or negative) and (ii) the type of action (False Alarms and Fast Hits) preceding word presentation as within-subject factors. We did not include in these analyses trials corresponding to Slow Hits because this action type did not lead to any significant and consistent evaluative priming effect in previous studies (Aarts et al., 2012, 2013), nor in the present one, $\mathrm{t}<1$. This may be due to the fact that the putative valence of slow hits (unlike either Fast Hits or False Alarms) was somehow ambivalent in the sense that a slow hit was a correct action in absolute terms but performed too slowly relative to the arbitrary response deadline (hence probably carrying also a negative connotation). Even though the dichotomy between Fast Hits and Slow Hits could appear somewhat arbitrary at first sight, earlier studies using the same speeded Go/noGo task already showed that these two trial types elicited different neurophysiological effects (at the CRN level specifically) and could be dissociated from one another (see Vocat et al., 2008; Aarts et al., 2013). Furthermore, auxiliary analyses (not reported here) performed on the RT distributions for the three main action types (False Alarms, Fast Hits and Slow Hits) revealed only partial overlap between Fast Hits and Slow Hits, suggesting that these two trial types actually corresponded to different behavioral outcomes in terms of RT speed during the speeded Go/noGo task (see Pourtois, 2011for a similar analysis and conclusion). Also, correct inhibitions were not included in the analysis because no overt action was performed in this 
condition. An auxiliary analysis showed that following correct rejections, the speed to categorize positive vs. negative words was balanced $(\mathrm{t}<1)$.

\section{Face affective priming task.}

Outliers. Correct hits with RTs shorter than $150 \mathrm{~ms}$ or longer than $500 \mathrm{~ms}$ during the Go/noGo task were discarded. With respect to Negative Faces, $17 \%$ of trials were removed, whereas $15 \%$ of Positive faces were removed in the Positive faces condition. Across all trial types, the removal amounted $15 \%$ in total. For the subsequent evaluative categorization task, trials were excluded if RT exceeded 2.5 SD above or below the mean reaction time computed per condition (Negative words: 3\%, Positive words: 4\%) on an individual level. Using the 2.5 SD criterion on a group level, the data of 2 participants (for accuracy) were removed from the subsequent statistical analyses. After exclusion, on average 54 trials and 53 trials were included to compute mean RTs for negative words and positive words after Negative faces, respectively. In the case of words following Positive faces, on average 55 trials and 55 trials were included for negative words and positive words, respectively ( $n=36$ participants). For accuracy $(n=34$ participants), on average 58 trials and 57 trials were included to compute mean performance for negative words and positive words after Negative faces, respectively. In case of words following Positive faces, on average 60 trials and 59 trials were included for negative words and positive words, respectively.

Face discrimination task. Accuracy and RTs for correct responses (i.e., correct presses on Go trials that did not exceed $400 \mathrm{~ms}$ ) were analyzed separately using paired samples T-tests. For accuracy, correct responses were defined as all correct key presses.

Evaluative categorization task. Accuracy and RTs for correct responses (i.e., correct presses on Go trials) were analyzed using ANOVAs as a function of (i) the valence of the face 
(positive or negative) and (ii) the valence of the target word (either positive or negative) preceding word presentation. Incorrect key presses during No Go trials and correct inhibitions during No Go trials were not included in this analysis.

\section{Relationship between Components of Anxiety and Evaluative Priming Effect. In}

order to determine whether the evaluative priming effect was influenced by anxiety (and more specifically whether either worry or arousal, or perhaps both components played a role), the priming effect for actions was calculated as follows: the magnitude of the evaluative priming was calculated as the RT difference between incongruent action-word pairs (i.e., False Alarms/positive and Fast Hits/negative) and congruent action-word pairs (i.e., False Alarms/negative and Fast Hits/positive) (see also Aarts et al., 2012 for a similar procedure). In the case of faces, congruent face-word pairs (i.e., negative face - negative word and positive face - positive word) were subtracted from incongruent face-word pairs (i.e., negative face - positive word and positive face - negative word). The larger this difference score, the stronger the priming effect. After these difference scores were obtained for each individual, the relationship between these scores and the specific anxiety scales were investigated by entering them as predictors in a stepwise regression analysis. Since the main hypothesis was to establish a differential (larger) weight to worry (PSWQ) when compared to arousal (MASQ-AA) as predictors of the action evaluative priming effect, only these two measures were included in the main model. Likewise, worry and arousal were also entered as main (and concurrent) predictors in the regression model used for the evaluative priming effect obtained for emotional faces.

Relationship between Components of Anxiety and PES. Individual RT scores for responding to positive and negative words after False Alarms were collapsed. Likewise, individual RT scores for responding to positive and negative words after Fast Hits were collapsed. Then the latter mean was subtracted from the former such that the larger this 
difference, the larger the PES effect. The (between-subjects) variability of the PES effect was then included in the stepwise regression analysis as dependent variable together with worry and arousal as predictor variables.

\section{Results}

\section{Action priming task.}

Go/noGo task. Participants made significantly less False Alarms $(M=75.48, S E=4.59)$ than Fast Hits $(M=115.11, S E=7.43), t(26)=-4.92, p<0.001,95 \%$ CI $[-59.18,-23.08]$ and had longer RTs for False Alarms $(M=219,44, S E=3,61)$ than for Fast Hits $(M=200,16, S E=$ $4,20), t(26)=8,25, p<0,001,95 \%$ CI [14.47, 24.09]. These results are compatible with previous findings obtained with the same Go/noGo task (Aarts et al., 2012, 2013).

\section{Evaluative categorization task.}

Speed. A test of normality (Shapiro-Wilk) showed that the RT data were not normally distributed. Accordingly, we log-transformed them first and performed subsequently paired samples T-tests. This analysis showed a significant difference between negative and positive words after False Alarms, $t(1,26)=-2.49, p<0.05$, while this difference did not reach significance following Fast Hits. The ANOVA performed on the mean RTs for correct responses (non log-transformed data) revealed a significant interaction effect between action type and word type, $F(1,26)=11.80, p<0.01, \eta^{2}=0.31$ (see Figure 2 ). More specifically, a paired samples Ttest revealed that RTs for negative words following False Alarms were shorter compared to RTs for positive words following False Alarms, $t(26)=-2.45, p<0.05,95 \%$ CI $[-53.77,-4.75]$. The opposite pattern was found for Fast Hits, in which RTs for negative words following Fast Hits were longer compared to RTs for positive words following Fast Hits. However, this latter difference did not reach significance, $t(26)=1.38, p=0.18,95 \%$ CI $[-5.16,26.18]$. The ANOVA did also reveal a significant main effect of action type, $F(1,26)=28.77, p<0.001, \eta^{2}=0.53$ : 
there were overall longer RTs for evaluating emotional words following False Alarms compared to words following Fast Hits, an effect in line with a systematic PES, as found in previous studies (Danielmeier \& Ullsperger, 2011; Rabbitt, 1966).

Accuracy. The ANOVA performed on accuracy data (i.e. \% correct responses) revealed only a significant main effect of action in which words following False Alarms were categorized less accurately than words following Fast Hits, $F(1,25)=7.91, p<0.01, \eta^{2}=0.24$ (Figure 2). The trend in categorizing negative words better than positive words following False Alarms was not significant, $t(25)=1.26, p=0.22$, while the symmetric effect following Fast Hits was not present.

\section{Relationship between worry, arousal and action priming task.}

Relationship with evaluative priming. We only focused on RT data, since the priming effect was absent for accuracy. A stepwise multiple regression was conducted to evaluate whether worry or arousal (or both) provided significant predictors of the evaluative priming effect. When worry was entered alone into the regression equation, it was significantly related to the evaluative priming effect $\mathrm{F}(1,25)=6.30, p<0.05$. The multiple correlation coefficient was 0.45 , indicating that approximately $20 \%$ of the variance of the evaluative priming effect could be accounted for by PSWQ scores. More specifically, PSWQ scores were negatively associated with the evaluative priming effect (see Figure 3), Unstandardized Beta $=-0.87, t(25)=-2.51, p<$ 0.05, CI 95\% [-1.59, -0.16]. By comparison, MASQ-AA scores did not relate to the evaluative priming effect $(t(25)=1.21, p=0.24)$.

Relationship with PES. When arousal was entered alone into the regression equation, it predicted significantly PES, $\mathrm{F}(1,25)=13.97, p<0.01$. The multiple correlation coefficient was 0.60, indicating approximately $36 \%$ of the variance of PES could be accounted for by MASQ-AA 
scores. More specifically, MASQ-AA scores were positively associated with PES (see Figure 3), unstandardized Beta $=3.12, t(25)=3.74, p<0.01$, CI 95\% [1.40, 4.84]. By comparison, PSWQ scores did not predict PES $(t(25)=-0.39, p=0.70)$.

\section{Face priming task.}

Face discrimination task. Participants were faster in responding to positive $(M=353.41$, $S E M=5.53)$ compared to negative faces $(M=359.34, S E M=5.24), t(35)=2.56, p<0.05,95 \%$ CI $[1.23,10.63]$. Furthermore, participants made more errors during the blocks in which they only had to respond to positive faces but had to withhold responding to negative faces $(M=0.87$, $S E M=0.01)$, compared to the other blocks where the task rule was reversed $(M=0.90, S E M=$ $0.01), t(35)=4.78, p<0.01,95 \% \mathrm{CI}[0.02,0.05]$. This indicates that inhibition to negative faces was less efficient than to positive faces.

\section{Evaluative categorization task.}

Speed. The ANOVA performed on the mean RTs for correct responses revealed a significant interaction effect between face type and word type, $F(1,35)=5.18, p<0.05, \eta^{2}=$ 0.13 (see Figure 4). Post-hoc paired samples T-tests did not reveal significant RT differences for discriminating negative relative to positive words after negative faces , $t(35)=-1.68, p=0.10$, $95 \%$ CI $[-25.50,2.40]$, or the symmetric effect following positive faces, $t(35)=0.82, p=0.42$, 95\% CI [-7.83, 18.46]. However, participants responded significantly slower to incongruent trials (negative words following Fast Hits and positive words following False Alarms; $M=556.01$, $S E M=12.34$ ) compared to congruent trials (negative words following False Alarms and positive words following Fast Hits; $M=547.58, S E M=13.21), t(35)=2.28, p<0.05,95 \%$ CI $[0.91$, 15.96]. The ANOVA did also reveal a significant main effect of face type, $F(1,35)=9.87, p<$ 
$0.01, \eta^{2}=0.22$ : there were overall longer RTs for words following negative faces compared to words following positive faces.

Accuracy. The ANOVA performed on accuracy data (i.e. \% correct responses) revealed no significant effects.

Relationship with the face evaluative priming effect. We only focused on RT data, since the priming effect was absent in the accuracy data. In doing so, a stepwise multiple regression was conducted to evaluate whether worry or arousal (or both) could predict the evaluative priming effect of faces. However, none of these two variables reached significance.

\section{Auxiliary analyses}

We performed additional analyses in which the criteria used for trial removal were less strict, namely an analysis in which the RT for arrows/faces was $100 \mathrm{~ms}-1000 \mathrm{~ms}$ and even 50 ms -1000 ms. The main statistical outcome (i.e., evaluative priming of actions, and modulation of this effect by worry) remained unchanged with $F$-values exceeding 11.20 and $p$-values $<0.01$ for the evaluative priming found in the Action affective priming task and the Face affective priming task. In case of the modulation of either worry or arousal on evaluative priming and PES, the effect of arousal on PES remained significant after the adoption of less strict criteria for trial exclusion $(F$-values $>7.56, p$-values $<0.01)$. For worry and evaluative priming, the effect remained significant with the use of the $100 \mathrm{~ms}-1000 \mathrm{~ms}$ criterion for trial exclusion $(F$-value $=$ $5.20, p$-value $=0.03)$, while it became marginally significant with the $50 \mathrm{~ms}-1000 \mathrm{~ms}$ criterion $(F$ - value $=3.52, p$-value $=0.07)$

In another analysis, we included the data of the two outliers (based on RT). Their inclusion did not alter the main statistical outcome however: for the Repeated Measures ANOVA, the two-way interaction effect between action and word remained significant, $F(1,28)$ 
$=10.55, p<0.01)$. Moreover, we still found significant correlations between priming and worry on the one hand $(r=-0.35, p<0.05)$, and PES and arousal on the other hand $(r=0.47, p<0.01)$, going in opposite directions.

\section{Experiment 2}

In Experiment 2, state worry was induced by means of an (unrelated) anagram task, while participants performed the same action priming task as described here above (Experiment 1). This way, we could assess whether state worry also undermined the rapid and automatic affective tagging of actions, like trait worry was found to do in Experiment 1.

\section{Method}

Participants. Fifty-two individuals took part in Experiment 2. Participants were randomly assigned to either the worry group or the control group. Eight participants were excluded from the subsequent statistical analysis, due to technical problems or excessively poor performance on the anagram task. Additionally, eleven participants (of which 8 originally belonged to the worry group and 3 belonged to the control group) were removed since they did not commit a sufficient number of False Alarms (i.e., minimum 10) to compute reliable accuracy or RT estimates for each condition separately (i.e., positive words following False Alarms vs. negative words following False Alarms). Of the remaining participants, 15 were part of the worry group, whereas 18 were included in the control group. The worry group consisted of 11 females whereas the control group consisted of 8 females. Since women are generally more likely to engage in rumination (Nolen-Hoeksema \& Jackson, 2001) and the relationship between performance monitoring and worry has been reported to be sex-specific (Moran, Taylor, \& Moser, 2012), we made sure that there was no asymmetry in gender distribution between these 
two groups, $\chi 2(1, \mathrm{~N}=33)=2.80, p=0.16$. Furthermore, the groups did not differ significantly in terms of age (worry group: $M=20, S D=2.28$; control group: $M=22, S D=4.23$ ), $F(1,31)=$ 2.48, $p=0.13$. All participants were native Dutch speakers. As a compensation, participants received either 10 euros or course credits for their participation.

\section{Stimuli and instruments.}

Tasks.

Action affective priming task. In this experiment, the same task was used as in Experiment 1.

Anagram task. Scrambled Dutch words were presented in black with a font size of 18 in the center of the screen one by one on a white homogenous background. All words were derived from the Dutch affective rating list of Hermans and De Houwer (1994). Since the aim of this experiment was to induce worry, we did not want to create any uncontrolled interference effect by using emotional words with this task. Therefore, we selected neutral words only. More specifically, we chose words that had a mean score on a Likert scale ranging from 1 (negative) to 7 (positive). We selected 12 (out of the 14) neutral words for which we knew they could all be easily unscrambled/solved by all participants. This was established by a pilot study in which 15 participants (none of whom took part in Experiment 2 or 1) were asked to solve the scrambled words and subsequently rate the difficulty of finding the solution on a Likert scale going from 1 (easy) to 5 (difficult). All participants were able to unscramble these 12 words. The average difficulty scores and affective scores of the words (Hermans \& De Houwer, 1994) can be found in Table 1.

Questionnaires and scales.

$P S W Q$. See Method's section Experiment 1.

STAI Trait. See Method's section Experiment 1. 
MZRI. In the Dutch version of the Momentary Ruminative Self-focus Inventory (Mor, Marchetti, \& Koster, 2013), participants have to indicate to what extent they believe that their (current) internal feelings are in accordance with the six statements provided by the questionnaire. These statements consist of feelings of rumination, such as 'At this moment I am thinking about my life’. Ratings range from 1-Completely Disagreeing to 7-Completely Agreeing. Afterwards, scores on the six individual questions are aggregated and can range between 6 (low state rumination) and 42 (high state rumination).

\section{Other measurements.}

Five VAS scales were included. Two of these VAS scales provide information about valence and the other that tackles arousal in relation to error commission. The other three VAS scales were specifically designed to explore a difference between the groups concerning how annoying they found it not to find the word's solution during the anagram task (from not annoying at all to extremely annoying), how negative the feedback was perceived (from weakly negative to very negative) and how distracted they were while performing the action affective priming task by the anagram task (from not distracted at all to very distracted). All participants had to answer to these statements by indicating it on a continuous line (ranging from 0 to 100).

Procedure. Participants were instructed that the experiment consisted of two tasks and that the aim of the experiment was to assess linguistic performance on both tasks. In line with the (alleged) information that it was a linguistic experiment, the action affective priming task was referred to as 'the word evaluation task' and the anagram task was referred to as the 'semantic task'. Furthermore, they received information about the order of the tasks, namely that the two tasks were interleaved and that they would first start with the word evaluation task and afterwards the semantic task. Additionally, they were told that these two tasks would repeat three times. 
The procedure of the action affective priming task was similar to Experiment 1 (see here above). However, in comparison to Experiment 1, test blocks consisted of 60 trials instead of 72 trials. For the anagram task, participants received instructions to unscramble as much words as possible given a limited amount of time ( 2 minutes). The first letter of the scrambled word was always the first letter of the solution. Participants used the keyboard to type, followed by the enter-key to submit their solution. The procedure only continued if the word was correct. If the solution entered was incorrect, the procedure looped back to the current unscrambled word and participants were asked to try again. Furthermore, they were informed that their performance on this task would eventually be compared with that of 80 "matched" participants or peers who previously completed this same task, and that they would receive feedback relative to the mean performance of this group (hence a social evaluative component was induced). Unlike what was told to participants, the anagram task only consisted of two parts that were interleaved with the action affective priming task. In the first part, participants had to solve five words whereas in the second part, they had to solve seven words. For all participants, the order of the words was the same. They were told that the first letter of the scrambled word was provided (see Table 1). After participants solved all words (either five or seven), they were confronted with the difficult and unsolvable word. In this case, the word was unsolvable, since the first letter provided was actually not the first letter of the (unscrambled) word and based on this information, it could never be solved. This last unsolvable word was accompanied by a countdown clock (starting with 40 seconds remaining) that appeared 20 seconds after word onset. When these 40 seconds elapsed, the word disappeared and they received bogus feedback regarding their performance in relation to the performance of 80 past participants. In the worry group, participants received feedback that they belonged to a minority that actually performed worse than this comparison group. In contrast, individuals in the control group received feedback that they belonged to a 
group (majority) that performed equally well as the comparison group (see Figure 5). This (bogus) feedback was presented for a fixed duration of 25 seconds.

The foreknowledge that the anagram task would repeat after some time led to the induction of worrisome or intrusive thoughts, especially in the worry group that received negative feedback regarding performance on this anagram task (see manipulation check results based on the VASes here below). Finally, participants filled in the PSWQ, MZRI and STAI Trait. Additionally, they scored the VAS scales.

\section{Analyses of Data}

Manipulation check and group comparison. Results for the PSWQ, STAI Trait, MZRI and the VAS scales were compared between the two groups by means of T-tests.

\section{Action affective priming task.}

Outliers. Trials with RTs shorter than $150 \mathrm{~ms}$ or longer than $500 \mathrm{~ms}$ during the Go/noGo task were discarded. With respect to False Alarms, $10.3 \%$ of trials were removed, whereas $8.4 \%$ of Fast Hits were removed. Across all trials, the removal amounted $6.4 \%$ in total. For the evaluative categorization task, trials were excluded if the RT of an individual exceeded 2.5 SD above or below his/her own mean reaction time computed per condition (Negative words: $3.5 \%$, Positive words: $3.8 \%$ ). Two participants were discarded from the RT analysis since on average they exceeded 2.5 SDs or were below 2.5 SDs of the condition mean for at least one of the four conditions (action $\mathrm{x}$ word valence). In terms of accuracy, one participant was removed from analysis.

After exclusion, on average 22 trials and 18 trials were included to compute mean RTs for negative words and positive words after False Alarms, respectively. In case of words following Fast Hits, on average 49 trials and 51 trials were included for negative words and positive words, respectively ( $n=31$ participants). For accuracy ( $n=32$ participants), on average 23 trials and 21 
trials were included to compute mean performance for negative words and positive words after False Alarms, respectively. In case of words following Fast Hits, on average 51 trials and 52 trials were included for negative words and positive words, respectively

Go/noGo task. Accuracy and RTs were analyzed separately using paired samples T-tests. All data were analyzed using SPSS (version 20).

Evaluative categorization task. Accuracy and RTs (for correct responses) were analyzed separately using a repeated measures ANOVA with (i) the valence of the target word (either positive or negative) and (ii) the type of action (False Alarms and Fast Hits) preceding word presentation as within-subject factors whereas group was entered as the between-subjects factor.

\section{Results}

Manipulation check and group comparison. Means and p-values can be found in Table 2. For this analysis, outliers (two participants for RTs and one for accuracy) were removed. The analysis revealed a significant group difference regarding the way they evaluated the feedback they received after the word unscramble task. More specifically, on a scale ranging from 0 to 100 , participants in the worry condition perceived the feedback as being more negative $(M=70.54)$ than participants who were in the control condition $(M=30.22), t(29)=-4.20, p<0.01$.

Importantly, participants in the worry condition also indicated to be significantly more distracted by the word unscramble task while performing the action affective priming task, $t(29)=-2.85, p$ $<0.01$. In terms of trait differences, no group difference was found regarding worry (PSWQ) or overall anxiety (STAI). No significant group difference was found in state rumination (MZRI) either.

\section{Action affective priming task.}

Go/noGo task. Participants made significantly less False Alarms $(M=47.97, S E=2.32)$ than Fast Hits $(M=106.94, S E=4.91), t(30)=-10.90, p<0.001,95 \%$ CI $[-70.02,-47.92]$, and 
had longer RTs for False Alarms $(M=228.25, S E=3.32)$ than for Fast Hits $(M=209.68, S E=$ 3.93), $t(30)=9.80, p<0,001,95 \%$ CI $[14.70,22.44]$. These results are compatible with previous findings obtained with the same Go/noGo task (Aarts et al., 2012, 2013; Aarts \& Pourtois, 2010).

\section{Evaluative Categorization task.}

Speed. A test of normality (Shapiro-Wilk) revealed that the data were normally distributed. The ANOVA performed on the mean RTs for correct responses revealed a significant interaction effect between action type and word type, $F(1,29)=15.33, p<0,001, \eta^{2}=0.35$. More specifically, a paired samples T-test revealed that RTs for negative words following False Alarms were shorter $(M=572.8, S E=17.55)$ compared to RTs for positive words following False Alarms $(M=611.6, S E=19.99), \mathrm{t}(30)=-3.14, \mathrm{p}<0.01, \mathrm{CI} 95 \%[-64.13,-13.55]$. The opposite pattern was found for Fast Hits, in which RTs for negative words following Fast Hits were longer compared to RTs for positive words following Fast Hits. However, this latter difference did not reach significance, $t(30)=1.65, p=0.11,95 \%$ CI $[-2.9,26.97]$. Moreover, the ANOVA did reveal a significant main effect of action type, $\mathrm{F}(1,29)=29.69, p<0,001, \eta^{2}=0.47$ : overall longer RTs for words following False Alarms compared to words following Fast Hits, an effect in line with a systematic PES (Danielmeier \& Ullsperger, 2011; Rabbitt, 1966). The three-way interaction effect manipulation $\mathrm{x}$ action $\mathrm{x}$ word was not significant, indicating that manipulation did not influence the evaluative priming effect (Figure 6). However, manipulation did interact with action, $F(1,29)=4.07, p=0.05, \eta^{2}=0.07$. More specifically, the difference in PES was stronger for individuals in the control condition $(M$ words after False Alarms $=606.95, S E=$ 23.33; $M$ words after Fast Hits $=539.80, S E=16.33$ ) compared to individuals in the worry condition $(M$ words after False Alarms $=571.80, S E=27.45 ; M$ words after Fast Hits $=540.92$, $S E=19.21)$ 
Accuracy. The ANOVA performed on accuracy data (i.e. \% correct responses) revealed a significant interaction effect between action type and word valence, $F(1,30)=15.95, p<0.001$, ,$\eta^{2}=0.34$. More specifically, paired samples T-tests revealed that participants were less accurate to categorize words as positive following False Alarms $(M=0.84, S E=0.02)$ compared to negative words following False Alarms $(M=0.94, S E=0.01), t(31)=3.78, p<0.001$, CI 95\% $[0.04,0.14]$, whereas the (opposite) difference was less pronounced (and not significant) following Fast Hits (negative words: $M=0.94, S E=0.01$; positive words: $M=0.95, S E=0.01$ ), $\mathrm{t}(31)=-0.49, p=0.63$, CI 95\% [-0.03, 0.02]. Furthermore, the main effect of action type reached significance, $F(1,30)=17.07, p<0.001, \eta^{2}=0.36$, indicating higher accuracy following Fast Hits compared to False Alarms. The main effect of word type was also significant, $F(1,30)=$ $8.49, p<0.001, \eta^{2}=0.20$, for which accuracy on negative words was higher than accuracy on positive words. The interaction effect between group and word valence was marginally significant, $F(1,30)=3.76, p=0.07, \eta^{2}=0.09$ (Figure 6).

\section{Discussion}

The aim of this study was twofold. First, we wanted to investigate in Experiment 1 whether trait worry, rather than the physiological or arousal component of anxiety, did weaken the evaluative priming effect for actions, in keeping with a recent behavioral study showing that trait anxiety, as broadly defined, actually dampened the "online" affective marking of actions (Aarts et al., 2012). Second, we sought to test whether modulatory effects exerted by worry onto action monitoring were either trait or state-related. To this aim, in Experiment 2, we induced worry by means of an anagram task, while worry was treated as a trait variable in Experiment 1 . The results of these two experiments replicate the findings previously put forward by Aarts et al. (2012, 2013). More specifically, in these two experiments, we found evidence for a significant interaction effect at 
the group level between the putative affective value of the action (correct/positive vs. incorrect/negative) and the valence of the subsequent emotional word (positive vs. negative). Participants were quicker at categorizing negative words after response errors (False Alarms) than positive words, with a symmetrical (albeit weaker) effect following Fast Hits (correct actions), suggesting that actions were automatically marked as either good or bad. Crucially, in Experiment 1, we found that worry (in contrast to arousal) decreased the magnitude of this action-based affective tagging. Interestingly, we could show that this selective effect of worry onto action monitoring was confined to this specific process, and could not be explained by a general tendency to influence and reduce evaluative priming in general. This was confirmed by showing the lack of modulatory effects of worry on evaluative priming triggered by emotional faces used as cues (standard or control evaluative priming task). Moreover, we found evidence for a trait, as opposed to state dependency of this effect because the temporary induction of worry (by means of an anagram task) did not yield the same effect (see Experiment 2). Finally, we also found evidence for a modulatory role of physiological anxiety (arousal) onto action monitoring, at the level of PES, selectively. Enhanced arousal was associated with enhanced slowing down in RT speed following error commission. Here below, we discuss the possible implications of our findings.

In Experiment 1, we found that a diminished evaluative priming effect for actions was associated with higher levels of trait worry, suggesting an impoverished affective tagging of actions in high worriers. The attention control theory (M. W. Eysenck, 1979) posits that anxiety may trigger a shift away from external task-relevant stimuli (and increase mind wandering or inward/internal attention). However, our new results do not suggest that anxiety (and more specifically worry) is associated with an overall attention impairment during the action-based evaluative priming task. On the contrary, we even found evidence in an auxiliary regression 
analysis for faster RTs for word discrimination as a function of worry in this task $(\beta=-1,65, p<$ 0.05); a result which may be consistent with a higher level of vigilance in high worriers (Mogg et al., 1987). More generally, this latter framework appears valuable to explain the blunted actionbased priming effect observed in Experiment 1 as a function of worry. Mogg et al. (1987) previously suggested with their vigilance avoidance hypothesis that after an initial heightened vigilance to potentially threatening events (Oathes, Squillante, Ray, \& Nitschke, 2010), avoidance of them is fostered in worry. In this context, Oathes, Siegle, and Ray (2011) previously found that (chronic) worriers were actually faster and more accurate in making valence categorization responses (for positive, negative and neutral stimuli) than low worriers. In addition, concurrent physiological recordings indicated that pupil diameter was reduced in high worriers after emotional stimulus presentation; an effect that the authors linked to the occurrence of emotional avoidance. Interestingly, when the emotional stimulus was personally relevant, high worriers even experienced a period of so-called "hyporesponsivity" (pupil diameter decreased below baseline), whereas low worriers maintained relatively large pupil sizes. Accordingly, in our study, we could argue that the affective tagging of actions in high worriers was reduced due to emotional avoidance. However, this conclusion awaits empirical validation because in different experimental contexts, anxiety or negative affect was previously found to increase (but not decrease) evaluative priming. For example, in the studies of Haberkamp, Schmidt, and Schmidt (2013) and Haberkamp and Schmidt (2014), larger priming effects were obtained for (phobic) individuals when primes were fear-related. Noteworthy, a number of major methodological differences between these earlier studies and the present one prevent us from performing systematic comparisons between them. Accordingly, whether avoidance per se or another process (e.g., hypervigilance to threatening events, including response errors) underlies 
the reduced affective tagging of actions seen in our study with increased levels of (trait) worry remains unclear.

Furthermore, because self-generated actions are presumably more relevant to an individual compared to the presentation of external emotional faces, worry and emotional avoidance impinged on the evaluative priming effects for actions selectively, but not for emotional faces used as cues in Experiment 1. This dissociation is in accordance with studies that already showed a larger impact of personally relevant stimuli or material on emotional processing in high compared to low worriers (Oathes et al., 2011; Siegle, Steinhauer, Carter, Ramel, \& Thase, 2003). Hence, although both tasks in Experiment 1 (i.e., the action affective priming task and the face affective priming task) had a similar procedure and time line and relied on affective primes each time, only the evaluative priming of actions was reliably diminished by worry presumably because of a heightened emotional avoidance for self-relevant primes.

As such, these new behavioural results (Experiment 1) provide further evidence for a modulatory role of anxious apprehension (worry), but not arousal, onto early stages of action monitoring, as previously advocated by Moser et al. (2013). These authors put forward the assumption that overactive early error monitoring processes seen in anxiety (at the level of the ERN component) primarily stem from worry. More generally, this dissociation also accords with recent neurobiological models and data emphasizing non-overlapping modulations and effects of worry vs. arousal in the human brain (Bijsterbosch et al., 2014). Accordingly, worry could negatively influence early stages of action monitoring taking place in the rostral cingulate zone (RCZ) and reflected by the ERN component (Koban \& Pourtois, 2014; Ullsperger et al., 2014), thereby undermining the normal action-based evaluative priming effect. Interestingly, Aarts et al. $(2012,2013)$ already provided indirect evidence for this conjecture by showing that trait anxiety reduced the action based priming effect, with effects measurable at the level of the ERN/CRN 
component. In light of the evidence gathered in the current study (Experiment 1), we would argue that worry is likely to be the critical component of trait anxiety underlying these modulations during action monitoring. Whether worry influences action monitoring via modulations of the RCZ only (Shackman et al., 2011), or via modulations arising in limbic structures (such as the amygdala) interconnected to the RCZ as well (Grupe \& Nitschke, 2013; Ochsner \& Gross, 2005; Pourtois et al., 2010; Stein, Simmons, Feinstein, \& Paulus, 2007) remains an open question for future research. Possibly, emotional avoidance accompanying worry dampens the normal amygdala functioning, as previously suggested in the case of Generalized Anxiety Disorder (Borkovec \& Inz, 1990; McClure et al., 2007; Nitschke et al., 2009). These effects triggered by worry in the amygdala might in turn influence action monitoring, given the strong and reciprocal anatomical connections between this limbic structure and the RCZ (Grupe \& Nitschke, 2013; Shackman et al., 2011).

Importantly, In Experiment 2 we failed to find evidence for an effect of state worry on the evaluative priming effect, suggesting that trait, but not state worry influences action monitoring, thereby revealing specific constrains on affective modulations during decision making processes. The lack of modulation by state worry on the action based evaluative priming effect in Experiment 2 was evidenced even though the worry induction turned out to be effective (i.e., participants in the experimental group reported to be more distracted by the filler/anagram task during the evaluative categorization task than the control group) and a significant action-word priming effect was shown (hence irrespective of worry). Interestingly, previous studies already emphasized that worry induction could influence specific cognitive functions or processes such as attention orienting towards threat-related stimuli (Oathes et al., 2010; Williams et al., 2014), however in individuals that already had high levels of trait worry or were prone to worry, respectively, confirming a role of more stable trait dispositions (or interactions between state and 
trait factors) in the case of worry in its propensity to interfere with cognitive processes. Accordingly, our new behavioral results are compatible with this interpretation, but they also extend these earlier findings by showing that trait, but not state worry, can influence early and automatic action monitoring processes.

Whereas worry interfered with the affective tagging of actions, we found evidence for the notion that the arousal component of anxiety actually facilitated the PES. The PES is usually taken as evidence for an unspecific orienting response (Sokolov, 1960), here to deviant and unwanted response errors (Danielmeier \& Ullsperger, 2011; Notebaert et al., 2009). Previous studies already linked physiological arousal (heart rate, pupil diameter, skin conductance or alpha power) to the PES (Carp \& Compton, 2009; Hajcak et al., 2003a; Wessel, Danielmeier, \& Ullsperger, 2011). Interestingly, in Experiment 2, we also found that the experimental group had a smaller PES than the control group, which might also be tentatively explained by an increase in (tonic) arousal in the former compared to the latter group of participants. By means of the worry induction, not only worry but also presumably (tonic) arousal was presumably augmented. Accordingly, the normal orienting response to the deviant response errors was less sharp or clearly expressed in this group of participants, compared to the control participants with no changes in worry (and presumably arousal). However, this interpretation is subject to caution given that we did not measure physiological arousal at the tonic level in Experiment 2.

In sum, the results of this study point to a functional dissociation between worry and arousal during action monitoring. While worry interferes with the rapid and automatic affective tagging (along a genuine valence dimension) of self-generated actions, arousal increases post-decision adjustment processes, at the level of the PES. Hence, these two components of anxiety appear to exert qualitatively different effects on two non-overlapping facets of action monitoring. Moreover, our results suggest that trait but not state worry influences early action monitoring 
processes. As such, these new findings add to the growing literature showing that anxiety is multifaceted and depending on its subtype or content (here with a focus on the distinction between worry and arousal), either facilitatory or interference effects can be evidenced during decision making.

\section{Acknowledgements}

This work is funded by the grant G015114N from the Research Foundation - Flanders (FWO).

\section{References}

Aarts, K., De Houwer, J., \& Pourtois, G. (2012). Evidence for the automatic evaluation of selfgenerated actions. Cognition, 124(2), 117-127. doi: 10.1016/j.cognition.2012.05.009

Aarts, K., De Houwer, J., \& Pourtois, G. (2013). Erroneous and Correct Actions Have a Different Affective Valence: Evidence From ERPs. Emotion, 13(5), 960-973. doi: $10.1037 / \mathrm{a} 0032808$

Aarts, K., \& Pourtois, G. (2010). Anxiety not only increases, but also alters early errormonitoring functions. Cognitive Affective \& Behavioral Neuroscience, 10(4), 479-492. doi: 10.3758/Cabn.10.4.479

Barron, E., Riby, L. M., Greer, J., \& Smallwood, J. (2011). Absorbed in Thought: The Effect of Mind Wandering on the Processing of Relevant and Irrelevant Events. Psychological Science, 22(5), 596-601. doi: 10.1177/0956797611404083

Berner, M. P., \& Maier, M. A. (2004). The direction of affective priming as a function of trait anxiety when naming target words with regular and irregular pronunciation. Experimental Psychology, 51(3), 180-190. doi: 10.1027/1618-3169.51.3.180

Bijsterbosch, J., Smith, S., Forster, S., John, O. P., \& Bishop, S. J. (2014). Resting state correlates of subdimensions of anxious affect. J Cogn Neurosci, 26(4), 914-926. doi: 10.1162/jocn_a_00512 
Bishop, S. J., \& Forster, S. (2013). Trait anxiety, neuroticism and the brain basis of vulnerability to affective disorder. . The Cambridge Handbook of Human Affective Neuroscience.

Borkovec, T. D., Alcaine, O., \& Behar, E. (2004). Avoidance theory of worry and generalized anxiety disorder. Generalized anxiety disorder: Advances in research and practice, 2004.

Borkovec, T. D., \& Inz, J. (1990). The Nature of Worry in Generalized Anxiety Disorder - a Predominance of Thought Activity. Behaviour Research and Therapy, 28(2), 153-158. doi: Doi 10.1016/0005-7967(90)90027-G

Botvinick, M. M., Braver, T. S., Barch, D. M., Carter, C. S., \& Cohen, J. D. (2001). Conflict monitoring and cognitive control. Psychological Review, 108(3), 624-652. doi: 10.1037//0033-295X.108.3.624

Brown, T. A., Antony, M. M., \& Barlow, D. H. (1992). Psychometric properties of the Penn State Worry Questionnaire in a clinical anxiety disorders sample. Behaviour research and therapy, 30(1), 33-37.

Bush, G., Luu, P., \& Posner, M. I. (2000). Cognitive and emotional influences in anterior cingulate cortex. Trends in Cognitive Sciences, 4(6), 215-222.

Carp, J., \& Compton, R. J. (2009). Alpha power is influenced by performance errors. Psychophysiology, 46(2), 336-343. doi: 10.1111/j.1469-8986.2008.00773.x

Clark, L. A., \& Watson, D. (1991). Tripartite Model of Anxiety and Depression - Psychometric Evidence and Taxonomic Implications. Journal of Abnormal Psychology, 100(3), 316336. doi: Doi 10.1037/0021-843x.100.3.316

Critchley, H. D., Elliott, R., Mathias, C. J., \& Dolan, R. J. (2000). Neural activity relating to generation and representation of galvanic skin conductance responses: A functional magnetic resonance imaging study. Journal of Neuroscience, 20(8), 3033-3040.

Danielmeier, C., \& Ullsperger, M. (2011). Post-error adjustments. Frontiers in Psychology, 2. doi: Artn 233 10.3389/Fpsyg.2011.00233

Defares, P. B., van der Ploeg, H. M., \& Spielberger, C. D. (1979). Zelf-behoordelings vragenlijst. Lisse: Swets and Zeitlinger.

Egloff, B., \& Hock, M. (2001). Interactive effects of state anxiety and trait anxiety on emotional Stroop interference. Personality and Individual Differences, 31(6), 875-882. doi: Doi 10.1016/S0191-8869(00)00188-4 
Endrass, T., \& Ullsperger, M. (2014). Specificity of performance monitoring changes in obsessive-compulsive disorder. Neuroscience and Biobehavioral Reviews, 46, 124-138. doi: 10.1016/j.neubiorev.2014.03.024

Eysenck, M. W. (1979). Anxiety, Learning, and Memory - Reconceptualization. Journal of Research in Personality, 13(4), 363-385. doi: Doi 10.1016/0092-6566(79)90001-1

Eysenck, M. W., Derakshan, N., Santos, R., \& Calvo, M. G. (2007). Anxiety and cognitive performance: attentional control theory. Emotion, 7(2), 336-353.

Eysenck, S. B. G., Eysenck, H. J., \& Barrett, P. (1985). A Revised Version of the Psychoticism Scale. Personality and Individual Differences, 6(1), 21-29. doi: Doi 10.1016/01918869(85)90026-1

Fazio, R. H., Sanbonmatsu, D. M., Powell, M. C., \& Kardes, F. R. (1986). On the Automatic Activation of Attitudes. Journal of Personality and Social Psychology, 50(2), 229-238. doi: Doi 10.1037//0022-3514.50.2.229

Forster, S., Elizalde, A. O. N., Castle, E., \& Bishop, S. J. (2015). Unraveling the Anxious Mind: Anxiety, Worry, and Frontal Engagement in Sustained Attention Versus Off-Task Processing. Cerebral Cortex, 25(3), 609-618. doi: 10.1093/cercor/bht248

Glaser, J., \& Banaji, M. R. (1999). When fair is foul and foul is fair: Reverse priming in automatic evaluation. Journal of Personality and Social Psychology, 77(4), 669-687. doi: Doi 10.1037/0022-3514.77.4.669

Grupe, D. W., \& Nitschke, J. B. (2013). Uncertainty and anticipation in anxiety: an integrated neurobiological and psychological perspective. Nature Reviews Neuroscience, 14(7), 488501. doi: 10.1038/nrn3524

Haberkamp, A., Schmidt, F., \& Schmidt, T. (2013). Rapid visuomotor processing of phobic images in spider- and snake-fearful participants. Acta Psychologica, 144(2), 232-242. doi: 10.1016/j.actpsy.2013.07.001

Haberkamp, A., \& Schmidt, T. (2014). Enhanced visuomotor processing of phobic images in blood-injury-injection fear. Journal of Anxiety Disorders, 28(3), 291-300. doi: 10.1016/j.janxdis.2014.02.001

Hajcak, G., McDonald, N., \& Simons, R. F. (2003a). Anxiety and error-related brain activity. Biological Psychology, 64(1-2), 77-90. 
Hajcak, G., McDonald, N., \& Simons, R. F. (2003b). To err is autonomic: error-related brain potentials, ANS activity, and post-error compensatory behavior. Psychophysiology, 40(6), 895-903.

Hermans, D., \& De Houwer, J. (1994). Affective and subjective familiarity ratings of 740 Dutch words. Psychologica Belgica, 34, 115-140.

Hermans, D., De Houwer, J., \& Eelen, P. (1994). The Affective Priming Effect - Automatic Activation of Evaluative Information in Memory. Cognition \& Emotion, 8(6), 515-533. doi: Doi 10.1080/02699939408408957

Jones, A. D., Cho, R. Y., Nystrom, L. E., Cohen, J. D., \& Braver, T. S. (2002). A computational model of anterior cingulate function in speeded response tasks: Effects of frequency, sequence, and conflict. Cognitive Affective \& Behavioral Neuroscience, 2(4), 300-317. doi: Doi 10.3758/Cabn.2.4.300

Keogh, E., \& Reidy, J. (2000). Exploring the factor structure of the Mood and Anxiety Symptom Questionnaire (MASQ). Journal of Personality Assessment, 74(1), 106-125. doi: Doi 10.1207/S15327752jpa740108

Koban, L., \& Pourtois, G. (2014). Brain systems underlying the affective and social monitoring of actions: An integrative review. Neuroscience and Biobehavioral Reviews, 46, 71-84. doi: 10.1016/j.neubiorev.2014.02.014

Lang, P. J., Greenwald, M. K., Bradley, M. M., \& Hamm, A. O. (1993). Looking at Pictures Affective, Facial, Visceral, and Behavioral Reactions. Psychophysiology, 30(3), 261-273. doi: DOI 10.1111/j.1469-8986.1993.tb03352.x

Lundqvist, D., Flykt, A., \& Öhman, A. (1998). The Karolinska directed emotional faces (KDEF). CD ROM from Department of Clinical Neuroscience, Psychology section, Karolinska Institutet, 91-630.

Maier, M. A., Berner, M. P., \& Pekrun, R. (2003). Directionality of affective priming: Effects of trait anxiety and activation level. Experimental Psychology, 50(2), 116-123. doi: $10.1027 / / 1618-3169.50 .2 .116$

McClure, E. B., Monk, C. S., Nelson, E. E., Parrish, J. M., Adler, A., Blair, R. J. R., . . Pine, D. S. (2007). Abnormal attention modulation of fear circuit function in pediatric generalized anxiety disorder. Archives of General Psychiatry, 64(1), 97-106. doi: DOI 10.1001/archpsyc.64.1.97 
Meyer, T. J., Miller, M. L., Metzger, R. L., \& Borkovec, T. D. (1990). Development and Validation of the Penn State Worry Questionnaire. Behaviour Research and Therapy, 28(6), 487-495. doi: Doi 10.1016/0005-7967(90)90135-6

Mogg, K., Weinman, J., \& Mathews, A. (1987). Memory Bias in Clinical Anxiety. Journal of Abnormal Psychology, 96(2), 94-98. doi: Doi 10.1037//0021-843x.96.2.94

Molina, S., \& Borkovec, T. D. (1994). The Penn State Worry Questionnaire: Psychometric properties and associated characteristics.

Moors, A., De Houwer, J., Hermans, D., Wanmaker, S., van Schie, K., van Harmelen, A. L., . . . Brysbaert, M. (2013). Norms of valence, arousal, dominance, and age of acquisition for 4,300 Dutch words. Behavior Research Methods, 45(1), 169-177. doi: 10.3758/s13428012-0243-8

Mor, N., Marchetti, I., \& Koster, E. H. (2013). The momentary ruminative self-focus inventory (MRSI): Validation and psychometric evaluation. Manuscript submitted for publication.

Moran, T. P., Taylor, D., \& Moser, J. S. (2012). Sex moderates the relationship between worry and performance monitoring brain activity in undergraduates. International Journal of Psychophysiology, 85(2), 188-194. doi: 10.1016/j.ijpsycho.2012.05.005

Moser, J. S., Moran, T. P., Schroder, H. S., Donnellan, M. B., \& Yeung, N. (2013). On the relationship between anxiety and error monitoring: a meta-analysis and conceptual framework. Frontiers in Human Neuroscience, 7. doi: Artn 46 10.3389/Fnhum.2013.00466

Nitschke, J. B., Sarinopoulos, I., Oathes, D. J., Johnstone, T., Whalen, P. J., Davidson, R. J., \& Kalin, N. H. (2009). Anticipatory Activation in the Amygdala and Anterior Cingulate in Generalized Anxiety Disorder and Prediction of Treatment Response. American Journal of Psychiatry, 166(3), 302-310. doi: 10.1176/appi.ajp.2008.07101682

Nolen-Hoeksema, S., \& Jackson, B. (2001). Mediators of the gender difference in rumination. Psychology of Women Quarterly, 25(1), 37-47. doi: Doi 10.1111/1471-6402.00005

Notebaert, W., Houtman, F., Van Opstal, F., Gevers, W., Fias, W., \& Verguts, T. (2009). Posterror slowing: An orienting account. Cognition, 111(2), 275-279. doi: 10.1016/j.cognition.2009.02.002

Oathes, D. J., Siegle, G. J., \& Ray, W. J. (2011). Chronic Worry and the Temporal Dynamics of Emotional Processing. Emotion, 11(1), 101-114. doi: 10.1037/a0021781 
Oathes, D. J., Squillante, C. M., Ray, W. J., \& Nitschke, J. B. (2010). The Impact of Worry on Attention to Threat. Plos One, 5(10). doi: ARTN e13411 10.1371/journal.pone.0013411

Ochsner, K. N., \& Gross, J. J. (2005). The cognitive control of emotion. Trends in Cognitive Sciences, 9(5), 242-249.

Peterson, R. A., \& Reiss, S. (1987). Test manual for the anxiety sensitivity index. Orland Park (IL): International Diagnostic Systems.

Pourtois, G. (2011). Early Error Detection Predicted by Reduced Pre-response Control Process: An ERP Topographic Mapping Study. Brain Topography, 23(4), 403-422. doi: $10.1007 / \mathrm{s} 10548-010-0159-5$

Pourtois, G., Vocat, R., N'Diaye, K., Spinelli, L., Seeck, M., \& Vuilleumier, P. (2010). Errors recruit both cognitive and emotional monitoring systems: simultaneous intracranial recordings in the dorsal anterior cingulate gyrus and amygdala combined with fMRI. Neuropsychologia, 48(4), 1144-1159.

Rabbitt, P. M. A. (1966). Errors and Error Correction in Choice-Response Tasks. Journal of Experimental Psychology, 71(2), 264-\&. doi: Doi 10.1037/H0022853

Ridderinkhof, K. R., Nteuwenhuis, S., \& Braver, T. S. (2007). Medial frontal cortex function: An introduction and overview. Cognitive Affective \& Behavioral Neuroscience, 7(4), 261 265. doi: Doi 10.3758/Cabn.7.4.261

Shackman, A. J., Salomons, T. V., Slagter, H. A., Fox, A. S., Winter, J. J., \& Davidson, R. J. (2011). The integration of negative affect, pain and cognitive control in the cingulate cortex. Nature Reviews Neuroscience, 12(3), 154-167. doi: 10.1038/nrn2994

Siegle, G. J., Steinhauer, S. R., Carter, C. S., Ramel, W., \& Thase, M. E. (2003). Do the seconds turn into hours? Relationships between sustained pupil dilation in response to emotional information and self-reported rumination. Cognitive Therapy and Research, 27(3), 365382. doi: Doi 10.1023/A:1023974602357

Simons, R. F. (2010). The way of our errors: Theme and variations. Psychophysiology, 47(1), 114. doi: 10.1111/j.1469-8986.2009.00929.x

Sokolov, E. N. (1960). Neuronal models and the orienting reflex. The central nervous system and behavior Research Methods, 187-276. 
Stein, M. B., Simmons, A. N., Feinstein, J. S., \& Paulus, M. P. (2007). Increased amygdala and insula activation during emotion processing in anxiety-prone subjects. American Journal of Psychiatry, 164(2), 318-327. doi: DOI 10.1176/appi.ajp.164.2.318

Taylor, S. F., Stern, E. R., \& Gehring, W. J. (2007). Neural systems for error monitoring: recent findings and theoretical perspectives. Neuroscientist, 13(2), 160-172.

Ullsperger, M., Fischer, A. G., Nigbur, R., \& Endrass, T. (2014). Neural mechanisms and temporal dynamics of performance monitoring. Trends in Cognitive Sciences, 18(5), 259267. doi: 10.1016/j.tics.2014.02.009

Vocat, R., Pourtois, G., \& Vuilleumier, P. (2008). Unavoidable errors: a spatio-temporal analysis of time-course and neural sources of evoked potentials associated with error processing in a speeded task. Neuropsychologia, 46(10), 2545-2555.

Watson, D., Weber, K., Assenheimer, J. S., Clark, L. A., Strauss, M. E., \& McCormick, R. A. (1995). Testing a tripartite model: I. Evaluating the convergent and discriminant validity of anxiety and depression symptom scales. Journal of abnormal psychology, 104(1), 3.

Wessel, J. R., Danielmeier, C., \& Ullsperger, M. (2011). Error Awareness Revisited: Accumulation of Multimodal Evidence from Central and Autonomic Nervous Systems. Journal of Cognitive Neuroscience, 23(10), 3021-3036. doi: DOI 10.1162/jocn.2011.21635

Williams, M. O., Mathews, A., \& Hirsch, C. R. (2014). Verbal worry facilitates attention to threat in high-worriers. Journal of Behavior Therapy and Experimental Psychiatry, 45(1), 8-14. doi: 10.1016/j.jbtep.2013.05.006

Yeung, N., Botvinick, M. M., \& Cohen, J. D. (2004). The neural basis of error detection: Conflict monitoring and the error-related negativity. Psychological Review, 111(4), 931-959. doi: 10.1037/0033-295x.111.4.931 


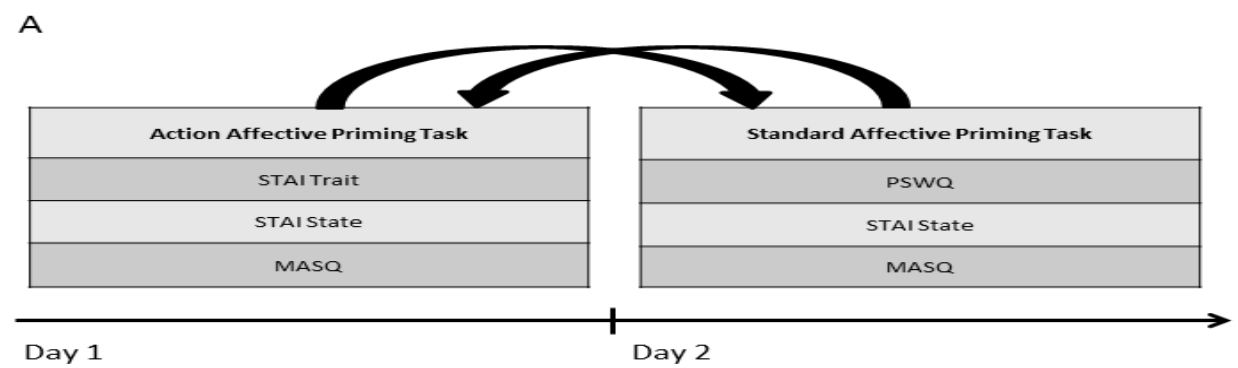

B

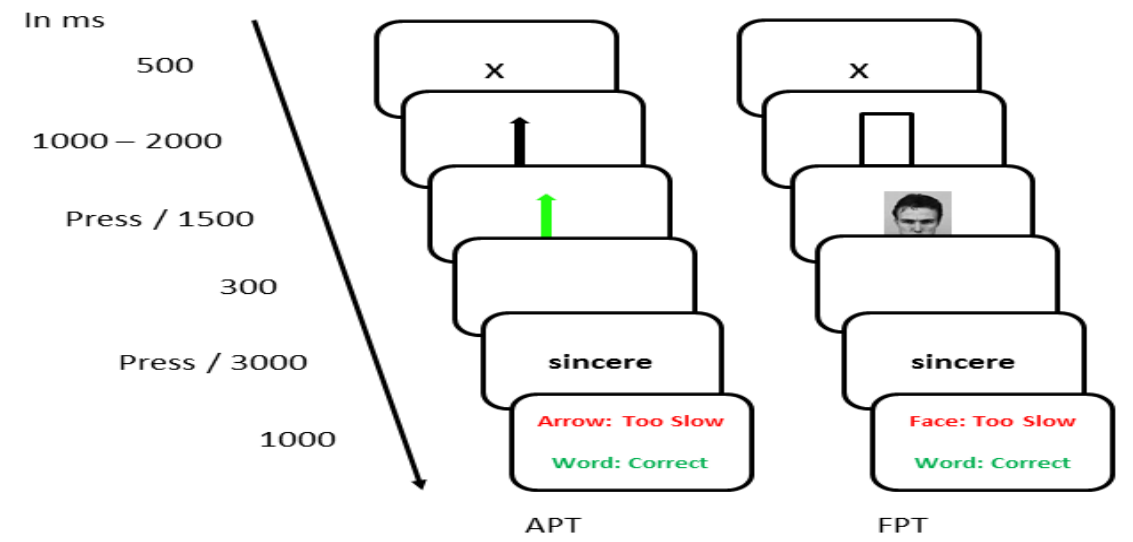

Figure 1. (A) Schematic representation of the two experimental sessions (Face affective priming task and Action affective priming task, see Experiment 1) that were held on two days, separated by one week. The order of the two tasks was counterbalanced across participants. To roughly equate the total duration of the two sessions, specific (trait-related) questionnaires were assigned either to the Face affective priming task (one session) or the Action affective priming task (the other one). By comparison, since STAI State and MASQ measure state-dependent effects, they were administered in both sessions. (B) Schematic 
representation of the experimental procedure (Experiment 1), showing the similarities and differences between the action priming task (APT) and the face priming task (FPT). These two tasks share a common time line and logic, consisting of a cue-target sequence. While cues are provided by correct or incorrect actions (to the go/nogo stimulus) in the APT, they comprise emotional faces in the case of the APT. Note that in both cases, the target (i.e., an emotional word that has to be categorized as fast as positive or negative) is the same.
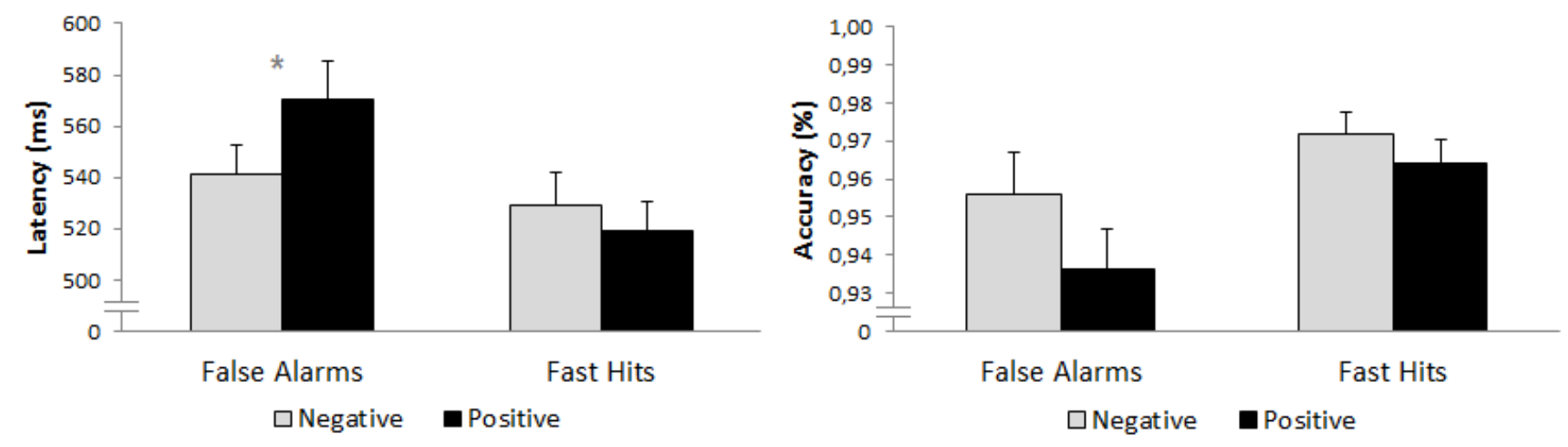

Figure 2. Results of Experiment 1 (Action affective priming task). (Left panel) mean RTs (+ 1 standard error of the mean - SEM bars) and (Right panel) mean accuracy (+ 1 standard error of the mean - SEM bars) for correct evaluative categorizations as a function of prime type (FAs or Fast Hits) and word type (Negative or Positive words)FAs. Asterisk indicates significant difference at $p<0.05$. 

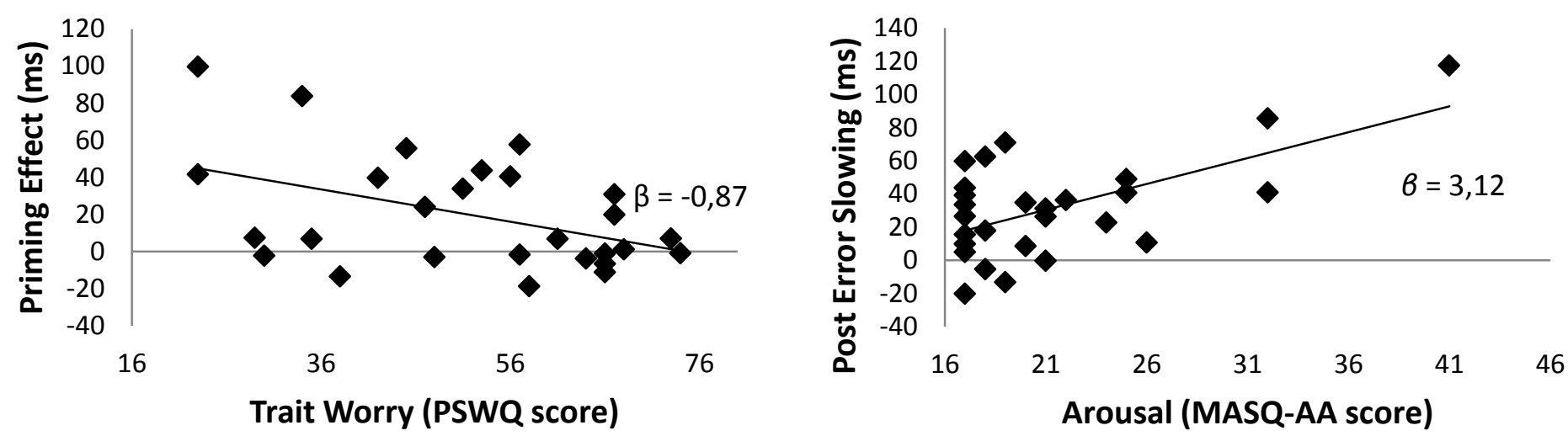

Figure 3. Results of Experiment 1 (Action affective priming task). (Left panel) Significant negative relationship between the evaluative priming effect and trait worry. (Right panel) In the case of arousal, there was a significant positive relationship with the PES. 


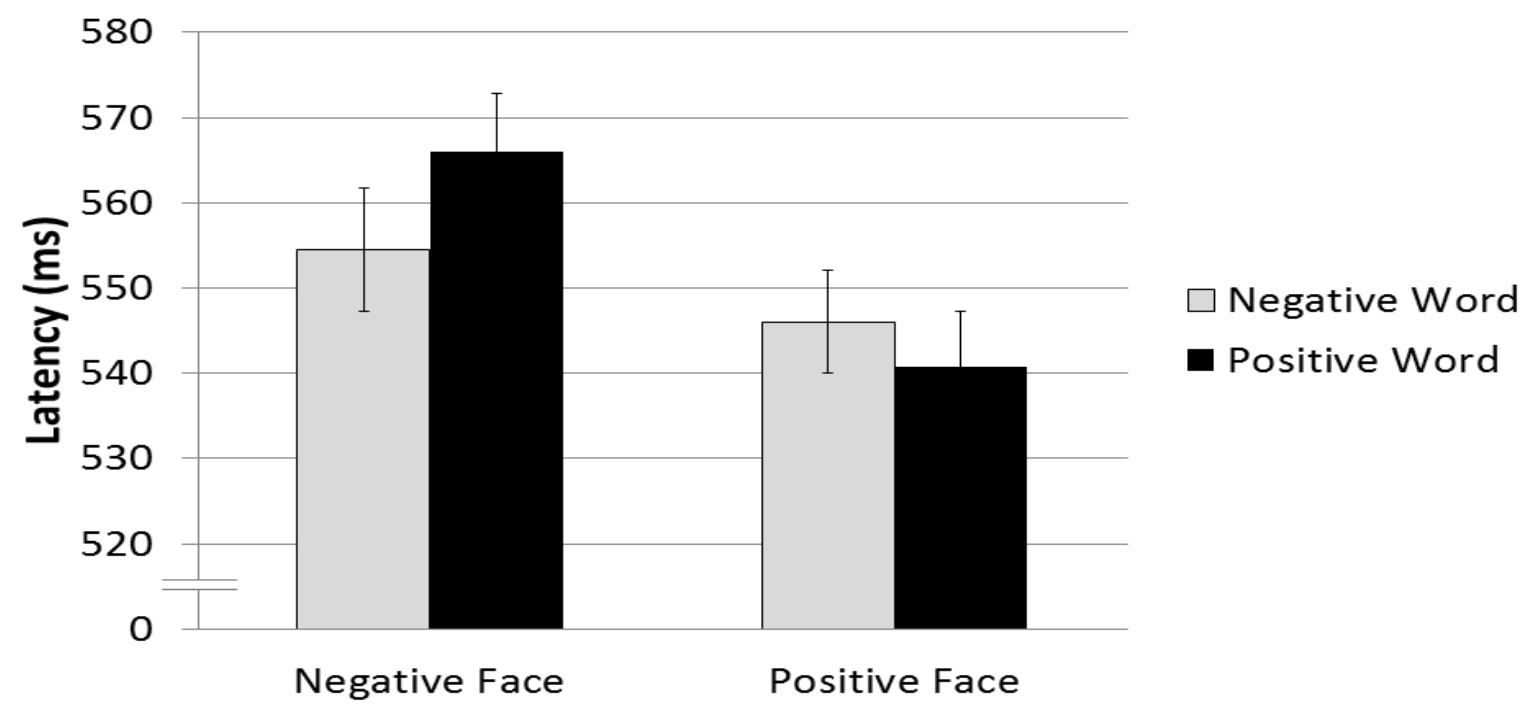

Figure 4. Results of Experiment 1 (Face affective priming task). Mean RTs (+ 1 SEM bars) for correct responses on Go-trials as a function of prime type (Negative face or Positive face) and word type (Negative or Positive words). Asterisk indicates significant difference at $p<0.05$. 
Table 1

Stimuli used in the anagram task (Experiment 2)

\begin{tabular}{lcccccc}
\hline & $\begin{array}{c}\text { Word } \\
\text { Order }\end{array}$ & $\begin{array}{c}\text { Dutch } \\
\text { Word }\end{array}$ & $\begin{array}{c}\text { Presented } \\
\text { Scrambled Word }\end{array}$ & $\begin{array}{c}\text { English } \\
\text { Translation }\end{array}$ & $\begin{array}{c}\text { Affective } \\
\text { score }\end{array}$ & $\begin{array}{c}\text { Difficulty } \\
\text { score }\end{array}$ \\
\hline Part 1 & & & & & & \\
Solvable words & 1 & Lijn & L NIJ & Line & 3.92 & 1 \\
& 2 & Doos & D OSO & Box & 4.13 & 1.07 \\
& 3 & Accent & A CECNT & Accent & 4.00 & 1.07 \\
& 4 & Papier & P EAIPR & Paper & 4.14 & 1.53 \\
Unsolvable word & 5 & Microscoop & M ISROOCCOP & Microscope & 4.00 & 1.28 \\
Part 2 & 6 & Agentschap & C SPNTAEAGH & Agency & 3.89 & \\
Solvable words & 1 & Disco & D ICSO & Disco & 4.09 & 1.00 \\
& 2 & Boog & B OGO & Bow & 4.00 & 1.13 \\
& 3 & Trompet & T RMPOET & Trumpet & 3.95 & 1.21 \\
& 4 & Gist & G SIT & Yeast & 3.97 & 1.67 \\
& 5 & Golf & G LFO & Golf & 4.17 & 1.07 \\
& 6 & Stoep & S OTEP & Sidewalk & 4.02 & 1.53 \\
& 7 & Vierkant & V AIEKRNT & Square & 4.00 & 2.33 \\
\hline
\end{tabular}

Note. In comparison to the solvable words, participants received bogus information regarding the first letter in case of the unsolvable word. For instance, for the solvable word lijn participants received information that $\mathrm{L}$ was the first letter and simply had to change the order of the other three characters. In case of the unsolvable word agentschap, participants also received information regarding the first letter. However, this was bogus information (first letter $\mathrm{C}$, whereas the word starts with an A). 
Results after processing

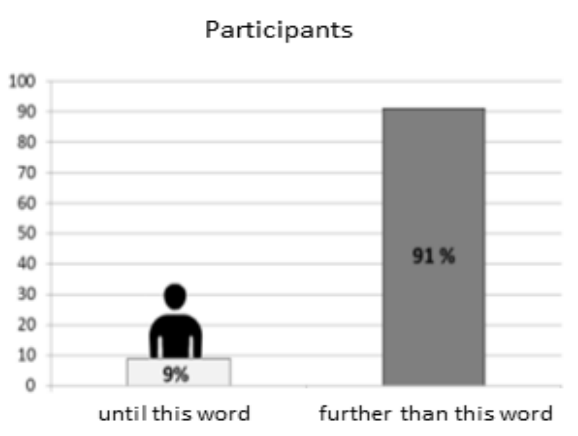

The group to which you are part of, is indicated by

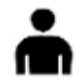

\section{Results after processing}

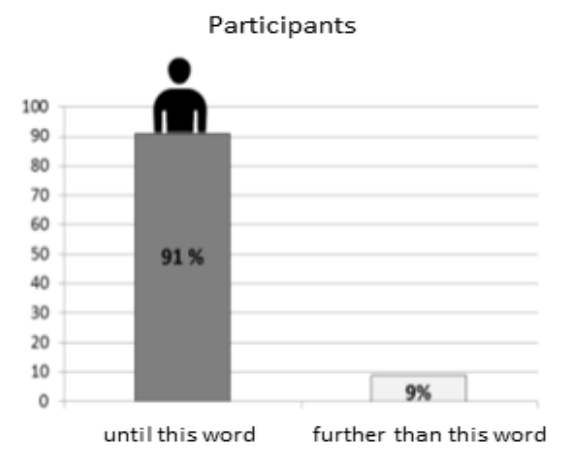

The group to which you are part of, is indicated by

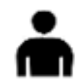

Figure 5. Feedback used in Experiment 2 during the anagram task. The left panel shows the feedback participants received in the worry condition whereas the right panel shows the feedback participants received in the control (no worry induction) condition. 
Table 2

Mean Differences between the Worry Group and the Control Group (Experiment 2)

\begin{tabular}{|c|c|c|c|c|c|c|}
\hline \multirow[b]{2}{*}{ Measure } & \multicolumn{2}{|c|}{ Control Group } & \multicolumn{2}{|c|}{ Worry Group } & \multirow{2}{*}{$F(1,28)$} & \multirow{2}{*}{$p$} \\
\hline & $M$ & $S E$ & $M$ & $S E$ & & \\
\hline PSWQ & 48.53 & 2.61 & 47.38 & 3.76 & .07 & .80 \\
\hline STAI Trait & 48.47 & 1.60 & 46.31 & 2.04 & .72 & .41 \\
\hline MZRI & 25.24 & 1.48 & 22.85 & 2.24 & .86 & .36 \\
\hline VAS Valence & 60.39 & 7.84 & 70.71 & 7.40 & .87 & .36 \\
\hline VAS Arousal & 61.32 & 7.51 & 42.23 & 9.29 & 2.61 & .12 \\
\hline VAS Word & 57.41 & 6.83 & 72.06 & 7.59 & 2.04 & .16 \\
\hline VAS Feedback & 30.43 & 6.39 & 70.54 & 7.63 & 16.44 & $<.01$ \\
\hline VAS Distraction & 24.47 & 5.18 & 53.08 & 7.56 & 10.42 & $<.01$ \\
\hline
\end{tabular}



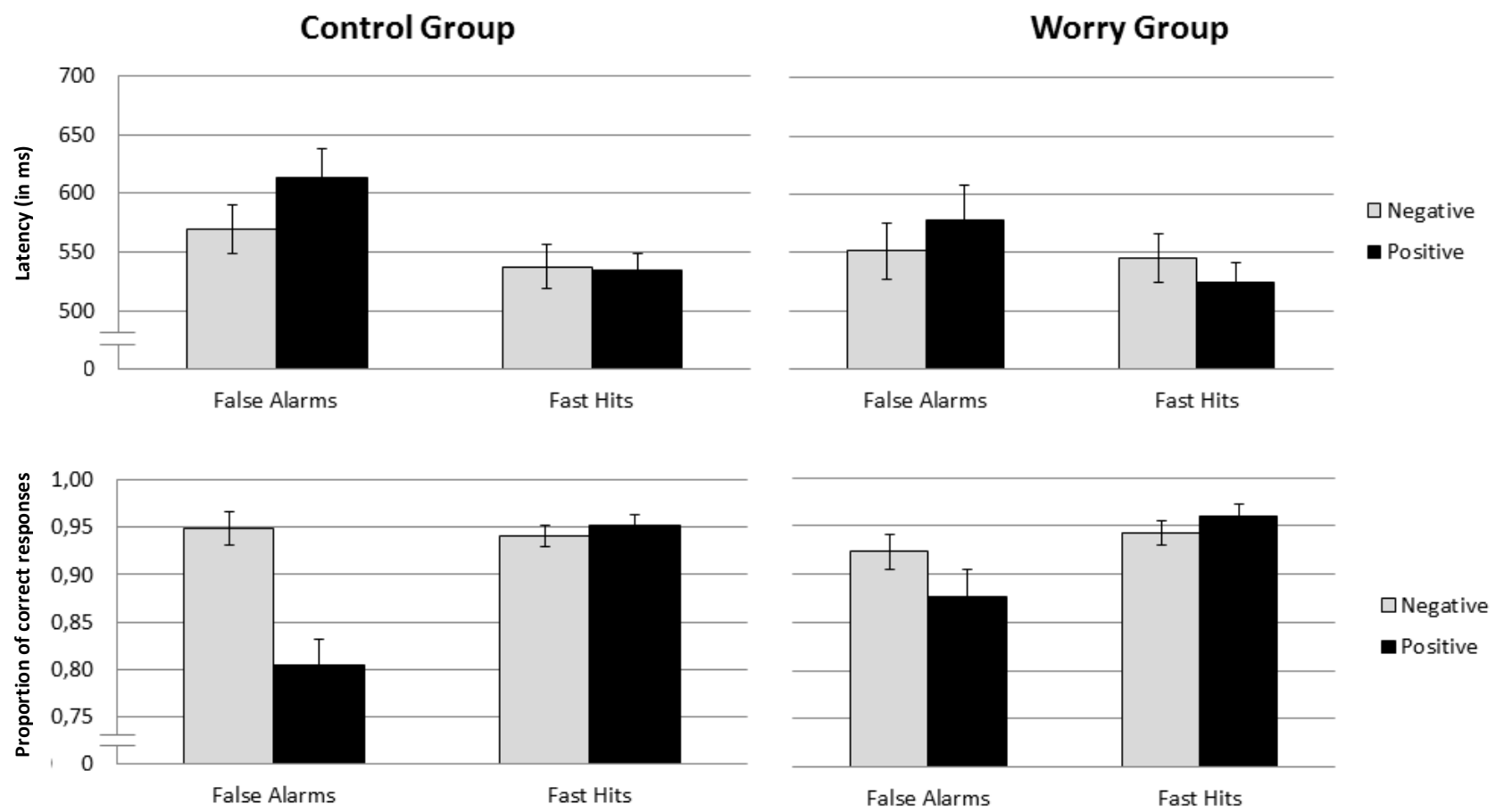

Figure 6. Mean RTs (+1 SEM bars; upper panel) and mean accuracy expressed in proportion of correct responses (+1 SEM bars; lower panel) for correct evaluative categorizations as a function of prime type (False Alarms or Fast Hits) and word type (Negative or Positive words), depicted for the control group and the worry group separately of Experiment 2. 